\title{
BILATERAL FALLOPIAN TUBE ADENOCARCINOMA
}

\author{
Anuradha Calicut Kini Rao ${ }^{1}$, Archana Shivamurthy², Vidya Monappa ${ }^{3}$
}

\section{HOW TO CITE THIS ARTICLE:}

Anuradha Calicut Kini Rao, Archana Shivamurthy, Vidya Monappa. "Bilateral Fallopian Tube Adenocarcinoma". Journal of Evolution of Medical and Dental Sciences 2015; Vol. 4, Issue 33, April 23; Page: 5757-5762,

DOI: $10.14260 /$ jemds/2015/841

ABSTRACT: Primary fallopian tube carcinoma [PFTC] is an uncommon, aggressive neoplasm. Diagnosis is based on ultrasonographic findings combined with a thorough histopathological examination. It must be considered as a differential diagnosis in patients presenting with vaginal bleed or discharge, with bilateral adnexal masses and those who fail to respond to antibiotic therapy and drainage. Tubal tuberculosis and trauma have been considered by some to be carcinogenic factors inducing multifocal tumorigenesis of the müllerian duct. Fallopian tube carcinoma being included as part of the cancer spectrum associated with inherited BRCA mutations. We present here a rare case report of a 49yr old lady who came with complains of white discharge per vagina of 6 months duration and underwent bilateral salpingo-oophorectomy, in view of nodular echogenic lesions in the bilateral adnexa on ultrasonography. A diagnosis of primary serous adenocarcinoma FIGO Stage IC was rendered after histopathological examination. It is important to note that metastasis to the tube is often bilateral in majority of the cases. Primary uterine and ovarian malignancies need to be excluded as in the present case.

KEYWORDS: Primary, fallopian tube, bilateral.

INTRODUCTION: Primary fallopian tube carcinoma is an extremely uncommon neoplasm, accounting for $0.14 \%-1.8 \%$ of all malignancies involving the female genital tract.[1] Bilateral involvement is even rare and seen in $10-20 \%$ cases.[2] Highly vulnerable, the fallopian tube is however, considered to be an organ of low oncogenic potential.[3] Clinically mistaken for a tuboovarian mass or an ovarian tumor the delayed onset of treatment contributes to the associated bad prognosis. It is often infrequently diagnosed prior to therapeutic intervention. ${ }^{[1,2]}$ Fallopian tube carcinoma has also been suggested to be included into the spectrum of tumors with inherited BRCA mutations. Commonly seen in postmenopausal women who are frequently asymptomatic, it can rarely present with vaginal bleeding, watery discharge, pain abdomen or pelvic mass. Nulliparous women appear to have a higher risk. It is also known to be associated with infertility and pelvic inflammatory disease.[2,3] Histologically it has resemblance to epithelial ovarian malignancies and distinguishing it from these tumors can be challenging at times. We report a rare case of an incidentally detected bilateral tubal adenocarcinoma occurring in a 49 year old lady.

CASE REPORT: A 49 year old female patient (gravida 1, para1) presented with history of white discharge per vagina of 6 months duration. She had regular menstrual cycles prior to the onset of menopause. There was no history of fever, abdominal pain or distension. No other significant medical and family history was obtained. On examination, cervix showed an unhealthy, bleeding ulcer on the posterior lip. The uterus was bulky and retroverted. Ultrasonography showed chronic cervicitis with pyometra and nodular echogenic lesion in bilateral adnexa. In view of the above findings and to evaluate further, hysterectomy with bilateral salpingo-oophorectomy was done. 


\section{CASE REPORT}

PATHOLOGICAL FINDINGS: On examination, both tubes were distended, tortuous with fusiform enlargement, the lumen being obliterated by a grey white friable growth with papillary excrescences. Histopathological examination showed the tumor in both the tubes to be composed of glands, fused papillae appearing as solid nests and papillary fronds lined by stratified epithelium with pleomorphic nuclei, few with prominent nucleoli, mitotic figures and scant cytoplasm. Focally few different patterns of arrangement of the tumor cells were observed. Thus a diagnosis of primary serous adenocarcinoma - FIGO (The International Federation of Gynecology and Obstetrics) Stage IC was rendered, after excluding uterine and ovarian primary tumors. [Figures 1, 2, 3, 4, 5, 6, 7]

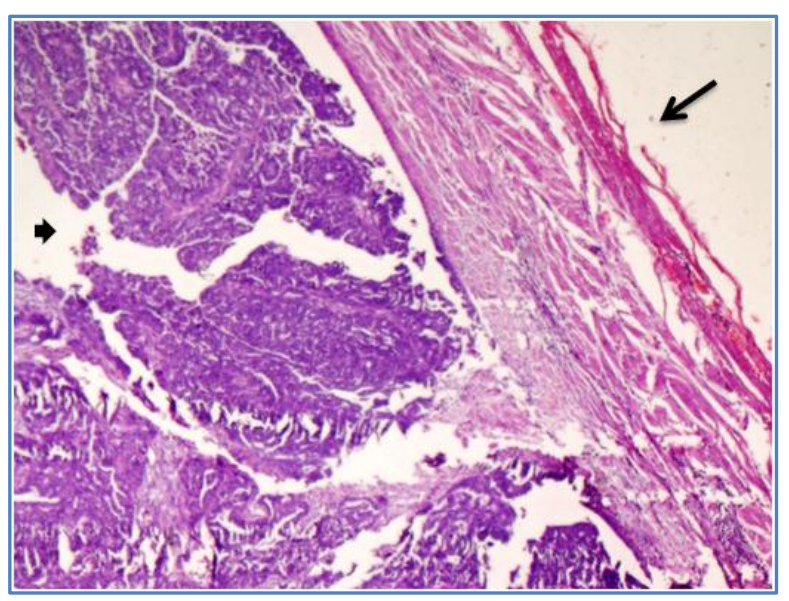

Fig. 1

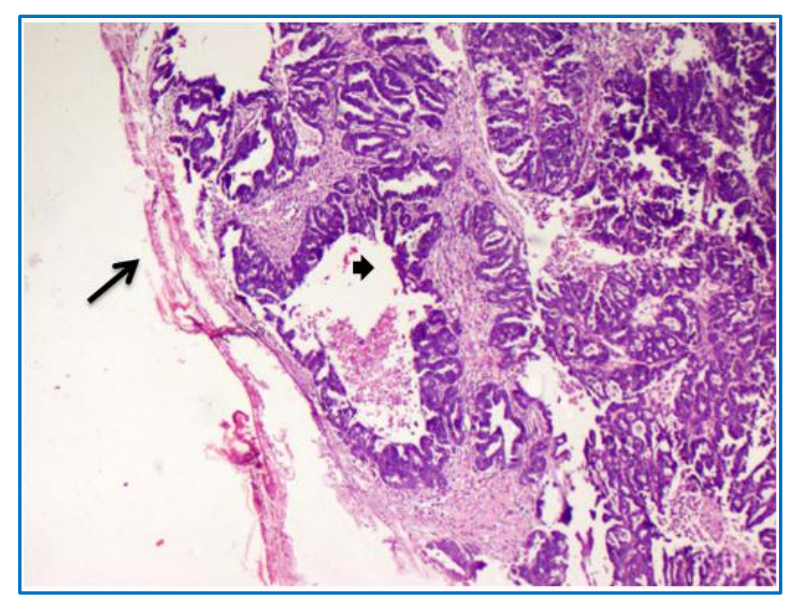

Fig. 2

Fig. 1 \& 2: Figures show adenocarcinoma involving the left and right tubes respectively. The long arrow shows the tubular wall, the short arrow indicates the tumor pattern - fused papillae appearing as solid nests in Fig 1 and glandular pattern in Fig. 2 [H \& E x40].

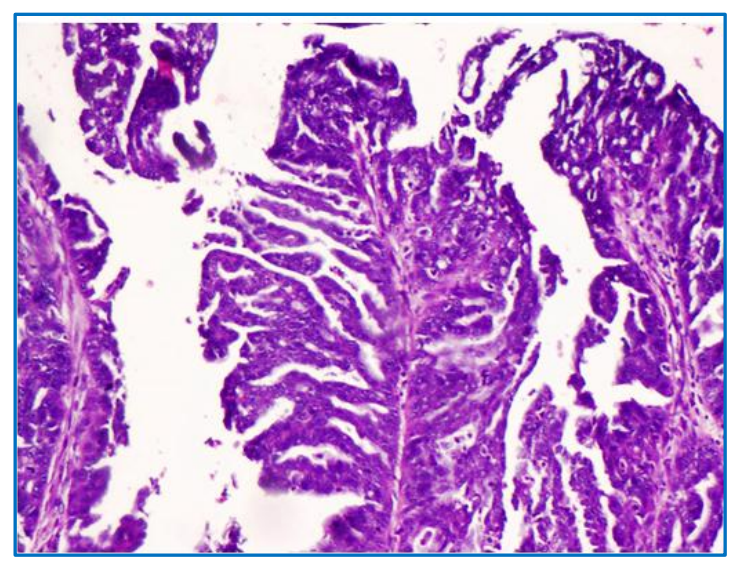

Fig. 3

Fig. 3: Figure shows tumor with papillary fronds with vascular core lined by malignant cells [H \& E $\mathrm{x} 100]$. 


\section{CASE REPORT}

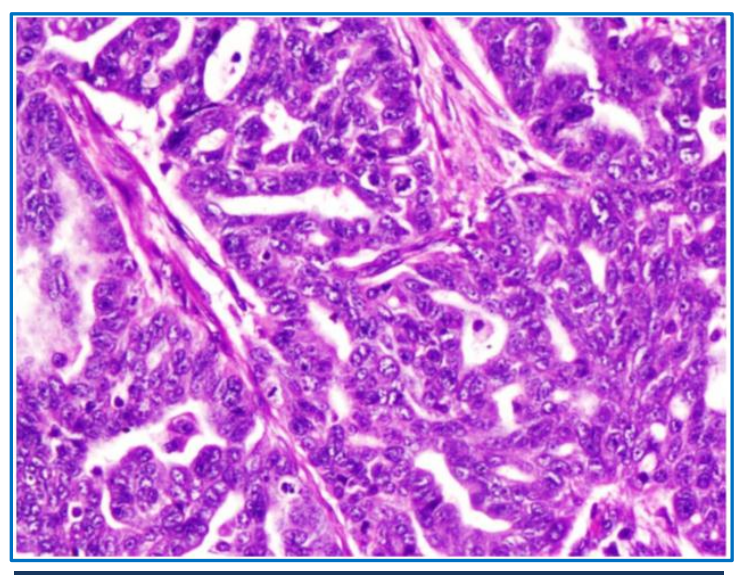

Fig. 4: Focal fused tubular pattern of the tumor cells [H \& E x100]

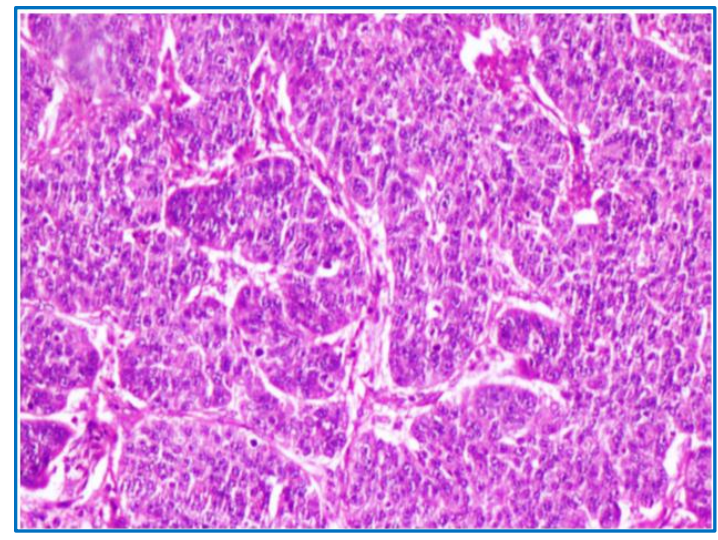

Fig. 6: Focal solid sheets of tumor cells [H\&E x100]

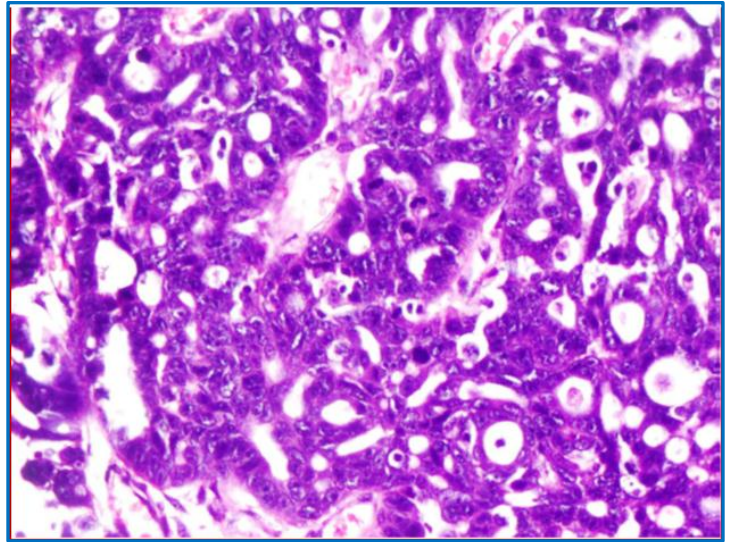

Fig. 5: Focal cribriform pattern of tumor cells [H\&E x100]

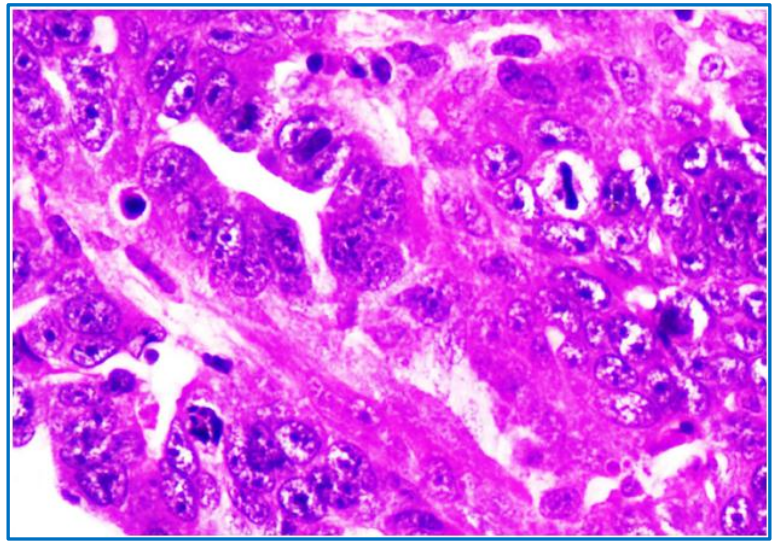

Fig. 7: High power view showing pleomorphic tumor cells and few mitotic figures [H\&E x400]

DISCUSSION: Primary adenocarcinoma of the fallopian tube was initially described by Renand in 1897.[2] It is the least common of all gynecologic malignancies, with incidence of about 3.6 per million women per year. The postulated histogenesis includes a relatively common mechanism of tubal tuberculosis and trauma considered as carcinogenic factors inducing multifocal tumorigenesis of müllerian duct neoplasms.[4,5] The most common histologic type is adenocarcinoma.[5] Usually unilateral and bilateral tumors have been reported following tubal sterilization. Chronic salpingitis is suggested to be a predisposing factor and germline mutation of BRCA 1 has been found with increased frequency in tubal carcinoma.[2] Nulliparous women are at a higher risk for developing primary fallopian tube carcinoma (PFTC) with parity being strongly protective.[6]

PFTC most frequently occurs in postmenopausal women between the fourth and sixth decades of life, with a median age of 55 years. However, it has been reported in young girls aged 17$19 \mathrm{yrs.}{ }^{[6,7]}$ In the present case the woman was 49 year old. The features considered pathognomonic of hydrops tubae profluens are intermittent discharge of clear or bloody fluid, either spontaneous or 
caused by pressure, followed by shrinkage of the adnexal mass.[1] However the classical clinical trial of tubal carcinoma includes Latzko's triad of symptoms, consisting of intermittent profuse serosanguinous vaginal discharge, colicky pain relieved by discharge, and abdominal or pelvic mass, reported in $15 \%$ of cases.[7] In the present case, the patient presented only with white discharge per vagina, adding least suspicion of a fallopian tube carcinoma.

A diagnosis of this entity with the help of vaginal cytology is also difficult as the cytological findings are positive in only $10-20 \%$ of cases..$^{[5]}$ Ren et al. suggested that the finding of dyskeratotic glandular cells on cervical smear should alert one regarding the possibility of fallopian tube carcinoma although these cells are seen in ovarian, serous and primary peritoneal carcinomas.[6] Resemblance to the ovarian carcinomas makes diagnosis challenging and even difficult.

A preoperative serum CA125 assessment is helpful, as it is elevated in $65 \%$ of PFTCs.[1] The ampullary portion of the fallopian tube is the usual site of origin of tubal carcinoma and its pattern of growth can be nodular, papillary, infiltrative, or massive. Fallopian tube carcinomas have a wide variety of gross appearances. These tumors can produce large amount of serous fluid leading a diffuse swelling and thus making the fallopian tube resemble a sausage. This picture often mimics intra-operative tubo ovarian abscess, hydro-, hemato-, or pyosalpinx. It can sometimes appear as solid tumor with or without papillary excrescences blossoming out of the tubal lumen. However it can be partly solid or cystic.[5] These tumors usually are confined to the tube. In the present case, we observed fusiform enlargement of both the tubes with papillary excrescences occupying the lumen.

Primary adenocarcinoma of the fallopian tube with papillary features is the most common histological type as seen in the present case. This type accounts for more than $90 \%$ of malignant tumors. Occasionally they have a dense inflammatory infiltrate that can simulate a salpingitis of non tuberculous type. Other less common histological types include clear cell carcinoma, squamous cell carcinoma, mixed carcinoma, endometrioid carcinoma, transitional cell carcinoma and sarcoma. Endometrioid type is usually less invasive and hence a favourable prognosis.[7,8]

The FIGO staging system assigns nearly two-thirds of patients to stage I or II and is based on surgical staging criteria similar to ovarian cancer. The present case was assigned FIGO stage IC. Fallopian tube carcinoma can metastasize via haematogenous, lymphatic, and peritoneal routes as well as have a direct extension. Even cerebral, bone and axillary lymph node metastasis has been reported. However, it is rare to find extra-abdominal metastases with minimal pelvic disease. Total abdominal hysterectomy with bilateral salphingo-oophorectomy is clearly the mainstay of treatment and probably the first approach to diagnosis. Patients with advanced disease (> Stage IIA) should be treated with post-operative combined chemotherapy with carboplatin plus paclitaxel. Some studies have also identified prognostic factors which include age of the patient, stage, advanced disease, residual tumor after initial surgery as important prognostic factors which can affect survival in these patients. $[3,9,10]$

CONCLUSION: The most important prognostic factors for outcome are stage of the disease during primary surgery and residual tumor. Of importance to the pathologist is that occult carcinoma in prophylactic salpingo-ophorectomy specimens may be found in the fallopian tube. Hence both tubes must be submitted and serially sectioned for microscopic evaluation. A preoperative differential diagnosis of PFTC should be considered for an adnexal mass with raised CA125 levels in all postmenopausal women and premenopausal women not responsive to antibiotic therapy for tubo- 
ovarian abscess. The importance of recognition lies in the higher rate of retroperitoneal and distant metastasis than associated in patients with epithelial ovarian cancer.

ACKNOWLEDGEMENTS: We wish to thank all the technical staff at Kasturba medical college, Manipal.

\section{REFERENCES:}

1. Jeung CI, Lee YS, Lee HN, Park EK. Primary Carcinoma of the Fallopian Tube: Report of Two Cases with Literature Review. Cancer Res Treat. 2009 June; 41 (2): 113-116.

2. Sarangthem B, Laishram S, Sharma AB, Konjengbam R, Debnath K. Primary bilateral tubal adenocarcinoma associated with uterine leiomyomas. Indian J Pathol Microbiol. 2008 Jan-Mar; 51 (1): 32-3. PMID: 18417847.

3. Jayashree K, Anubuti C, Sunila, Gundappa M. Primary fallopian tube adenocarcinoma with brain and lung metastasis. Indian J Pathol Microbiol 2009; 52: 596-8. doi: 10. 4103/0377-4929. 56148. PubMedPMID: 19805996.

4. N. A Healy, S. O Hynes, J. Bruzzi, S. Curran, M. O'Leary, and K. J Sweeney, "Asymptomatic Primary Fallopian Tube Cancer: An Unusual Cause of Axillary Lymphadenopathy, " Case Reports in Obstetrics and Gynecology, vol. 2011, Article ID 402127, 6 pages, 2011. doi: 10. $1155 / 2011 / 402127$.

5. Hosokawa C, Tsubakimoto M, Inoue $Y$, Nakamura T. Bilateral primary fallopian tube carcinoma: findings on sequential MRI. AJR Am J Roentgenol. 2006 Apr; 186 (4): 1046-50. DOI: 10. 2214/AJR. 05. 0491 PMID: 16554577.

6. Ren Z, Zhang YP, Yang HX, Zhu LR. Liquid-based cytology aids in primary fallopian tube cancer diagnosis. J Zhejiang Univ Sci B. 2009 Aug; 10 (8): 635-638. doi. 10. 1631/jzus. B0920040. PMCID PMC2722707.

7. Pectasides D, Pectasides E, Economopoulos T. Fallopian tube carcinoma: a review. Oncologist. 2006 Sep; 11 (8): 902-12. PubMed PMID: 16951394.

8. Inal MM, Hanhan M, Pllanci B, Tinar S. Fallopian tube malignancies: experience of Social Security Agency Aegean Maternity Hospital. Int J Gynecol Cancer 2004; 14: 595-99. PubMed PMID: 15304152.

9. Rakshit A, Dey R, Ranu Biswas R, Biswas SC. Primary Fallopian Tube Carcinoma (PFTC) - A Rare Genital Malignancy with Unusual Presentation. Al Ame en J Med Sci 2012; 5 (1): 95-97.

10. Courville XF, Cortés Z, Katzman PJ, Rosier RN. Case report: Bone metastases from fallopian tube carcinoma. Clin Orthop Relat Res. 2005 May; (434): 278-81. PubMed PMID: 15864065. 


\section{CASE REPORT}

\section{AUTHORS:}

1. Anuradha Calicut Kini Rao

2. Archana Shivamurthy

3. Vidya Monappa

\section{PARTICULARS OF CONTRIBUTORS:}

1. Professor, Department of Pathology, Kasturba Medical College, Manipal, Manipal University.

2. Assistant Professor, Department of Pathology, Melaka Manipal Medical College, Manipal, Manipal University.

FINANCIAL OR OTHER COMPETING INTERESTS: None
3. Associate Professor, Department of Pathology, Kasturba Medical College, Manipal, Manipal University.

\section{NAME ADDRESS EMAIL ID OF THE CORRESPONDING AUTHOR:}

Dr. Archana Shivamurthy, Department of Pathology, Basic Science Building, Manipal University Campus, Manipal. E-mail: archana_018@yahoo.co.in

Date of Submission: 28/02/2015.

Date of Peer Review: 03/03/2015. Date of Acceptance: 11/04/2015. Date of Publishing: 23/04/2015. 